\title{
An Entropy-based Clustering in Mobile Ad hoc Networks
}

\author{
Kimberly Robinson, Damla Turgut, and Mainak Chatterjee
}

\begin{abstract}
The distributiveness of mobile ad hoc networks makes resource allocation strategies very challenging since there is no central node to coordinate and monitor the activities of all the nodes in the network. Since a single node cannot be delegated to act as a centralized authority due to limitations in the transmission range, several delegated nodes may coordinate the activities in certain zones. This methodology is generally referred to as clustering and the nodes are called clusterheads. The clusterheads employ centralized algorithms in its cluster; however, the clusterheads themselves are distributive in nature.

In this paper, we propose a clustering method i.e., identify the clusterheads among all the nodes. Though there are several clustering algorithms that have been proposed; however, to the best of our knowledge, there is none that characterizes the different node parameters in terms of entropy. Entropy is a measure of information. We use the local information available to every node to determine the mutual information. We considered two parameters in the selection procedure, namely, energy and mobility. Extensive simulations have been conducted and the performance of the proposed clustering scheme has been shown in terms of the average number of clusterheads or clusters, the average number of cluster changes, and the average connectivity. The results demonstrate that the mutual information captured through entropy is very effective in determining the most suitable clusterheads.
\end{abstract}

\section{INTRODUCTION}

Deployment of infra-structured networks are time consuming and therefore cannot be set up at times of utmost emergency. Therefore, mobile multi-hop radio networks, also called ad hoc or peer-to-peer networks, play a critical role in places where a wired (central) backbone is neither available nor economical to build, such as law enforcement operations, battle field communications, disaster recovery situations, and so on. Such situations demand a network where all the nodes including the base stations are potentially mobile, and communication must be supported untethered between any two nodes. However, maintaining such seamless connection is difficult because of the inherent characteristic of mobile ad hoc networks i.e., highly dynamic topology changes due to the mobility of the nodes. Also, the bandwidth is limited and the signal quality is unpredictable.

In spite of these constraints, ad hoc networks are designed such that they are able to dynamically adapt themselves with the changing network configurations. One of the ways to handle the topology changes and maintain a connected network can be brought about by entrusting certain nodes with more responsibility. These nodes are typically called

Kimberly Robinson is a Senior Performance Engineer at the Corporate Technology Office, Adaptec, Inc. Orlando Technology Center, Florida; email: kimberly_robinson@adaptec.com.

Damla Turgut and Mainak Chatterjee are with the School of Electrical Engineering and Computer Science, University of Central Florida, Orlando, FL 32816-2450, email: \{turgut,mainak\} @cs.ucf.edu. clusterheads and are responsible for the formation of clusters each consisting of a number of ordinary nodes. A clusterhead is responsible for resource allocation to all the nodes belonging to its cluster. Due to the dynamic nature of the mobile nodes, their association and dissociation to and from clusters perturb the stability of the network and thus reconfiguration of clusterheads is unavoidable. This is an important issue since frequent clusterhead changes adversely affect the performance of other protocols such as scheduling, routing and resource allocation that rely on it. Choosing clusterheads optimally is an NP-hard problem [4]. Thus existing solutions to this problem are based on heuristic (mostly greedy) approaches and none attempts to retain the stability of the network topology [4], [7]. We believe a good clustering scheme should preserve its structure as much as possible when nodes are moving and/or the topology is changing. Otherwise, re-computation of clusterheads and frequent information exchange among the participating nodes will result in high computation overhead.

Several clustering algorithms and heuristics have been proposed in the literature [1], [6], [8], [9], [11], [12]. Many existing solutions take into account various heuristics of clusterhead suitability. However the most recognized ones are based upon clusterhead selection which rely on random events such as node id assignment (as in the Lowest ID algorithm) and the degree of connectivity (as in the Highest Degree algorithm). The Lowest ID [2], [3] heuristic assigns a unique ID to each node and chooses the node with the minimum ID as a clusterhead. Thus, the IDs of the neighbors of the clusterhead will be higher than that of the clusterhead. In Highest Degree [8], [10], each node broadcasts its ID to the nodes that are within its transmission range. A node $x$ is considered to be a neighbor of another node $y$ if $x$ lies within the transmission range of $y$. The node with maximum number of neighbors (i.e., maximum degree) is chosen as a clusterhead. If there is a tie, it can be broken arbitrarily by the nodes' IDs.

In this paper, we propose a distributed clustering algorithm which takes into consideration the local information available to all the nodes. This local information is measured in terms of entropy. We consider two parameters for the determination of the clusterheads- mobility of the nodes and their energy consumption. We show how we can compute the mutual information for these two factors and combine the results through a liner model. Our method of calculating the mutual information is generic enough and can easily be extended to include other physical factors. Through simulation experiments, we show the performance of our proposed scheme in terms of the average number of clusterheads, the average number of cluster changes, and the average connectivity. 
The rest of the paper is organized as follows. In section II, we discuss why a relative measure is required and how entropy can be used to capture the mutual information. In section III, we propose the entropy based clustering scheme considering mobility and energy of the nodes. The simulation model and results are presented in section IV. Conclusions are drawn in the last section.

\section{Motivation}

Different algorithms emphasize characteristics which may or may not be important based upon the architectural features of the individual network application. For example, a network that handles multimedia traffic, topological instability causes changes in the data transfer path threatening the timely transmission of streaming media. Thus heavy-duty clusterhead election techniques may be favored if greater network stability is achieved. In mobile ad hoc networks consisting of heterogeneous mobility devices, more powerful devices may overburden smaller less capable ones, placing a demand upon them which they are incapable of handling. These devices also may tend to transmit less data with less frequency, placing very little burden on the network. Certainly, an underpowered device would not be a good candidate as a clusterhead, regardless of its node ID; therefore, we conclude that the Lowest ID election in general is not a good algorithm for non-homogeneous networks. In highly mobile and dynamic networks, clusterhead elections are unavoidable, and the best course of action is to minimize the impact of the election process.

Algorithms which guarantee leader election with a certain amount of deviation from the optimal solution, would deem fit. That would also reduce the periodic instabilities brought on by the high rate of clusterhead changes, while minimizing the impact of the routing overhead associated with high levels of nodal re-affiliation.

Most algorithms work based on a pre-defined metric. The clustering decisions are based on the absolute values obtained by these metrics. Though it might seem to work, but at times the performance is misinterpreted. For example, consider an ad hoc network in operation. When the nodes are initialized, the performance of the network is expected to be at its best since the energy is at its maximum. However, with the lapse of time and energy depletion, there would also be a performance degradation. So, we must consider, the parameters at that point of time. More importantly, compare the nodes' suitability relative to each other.

We propose to take advantage of the mutual information; therefore, we use entropy based measures. Entropy has been widely used to capture the information content within a system. A measure of statistical dependence or correlation is usually sought between two or more parameters i.e., the random variables of a time series.

If $X$ and $Y$ are the random variables with joint distribution $p(X, Y)$ and marginal distributions $p(x)$ and $p(y)$, then the mutual information $I(X ; Y)$ is the relative entropy between the joint distribution and the product distribution. Hence,
$I(X ; Y)$ is given by

$$
I(X ; Y)=\sum_{x} \sum_{y} p(x, y) \log \frac{p(x, y)}{p(x) p(y)}
$$

\section{Proposed Algorithm}

By analyzing the relative entropy of the nodes, we can derive values which help determine nodal suitability. The proposed algorithm consists of the weighted linear sum of two entropy measurements: battery expectancy and mobility. We use these two parameters to demonstrate how the relative entropy can be calculated and the results combined to find the most suitable nodes to act as clusterheads. Though we use these two measurements, our algorithm is generic enough and can be extended to account for any other physical parameters.

\section{A. Mobility Entropy}

Determination of mobility entropy is based upon mutual information, which is an appropriate measure of change based upon previous expectation values. Each node collects a history of the broadcast (beacon) signals received from its neighbors during a period of time. Every node maintains a list containing the IDs of each node heard within the hearing range of the node. A node whose mobility is stable relative to its virtual cluster would see fewer changes in its neighborhood list. Since the motion is relative, it is impossible to ascertain whether the node itself or the neighbors node moved. Comparing the change in the neighbor list and more importantly the rate of change of the neighbor list, it is possible to infer the relative mobility of the nodes with respect to each other and to the clusterhead. We make a few probabilistic statements.

1) Several and frequent changes in neighbor list are more likely due to the node in question moving, rather than a large number of neighbors moving at once.

2) A few changes in neighbors is more likely due to neighbor movement away and towards the node, rather than the node's own movement.

3) Nodes which lie close to periphery of the transmission are likely to have a ping-pong effect i.e., in and out of the neighborhood list.

Observing the mobility of a node with respect to another, the probability that a node is moving, and the marginal probability that the node itself is moving can be calculated by the the mutual information.

Let us observe the behavior of the neighbor list of a particular node $i$ for a time interval of $\Delta t$. Let us assume that node $j$ appeared in the list at least once. We measure two quantities. First,the number of times node $j$ appeared. It can be noted that for a node to appear multiple times, it must also disappear that many times. Second, the total amount of time node $j$ stayed in the neighbor list of node $i$ during the interval $\Delta t$. The first quantity gives a measure of relative mobility and the second one provides an intuition about the relative stationarity of nodes $i$ and $j$. If a node $i$ counts the number of appearances of other nodes $j$, then it can compute the joint distribution for all the other nodes, i.e., 
$p(i, j)$ for all $j$. Also, $p(i)$ is known to node $i$, and it can gather information about $p(j)$ from its neighboring nodes, or the nodes that visited $i$. Thus we get the mutual information as was given by equation 1 .

\section{B. Energy Entropy}

Since clusterheads have the extra responsibility to forward packets on behalf of other nodes, they are prone to battery drainage. Therefore, a node with good residual battery power is a good candidate for being a clusterhead. Though, the remaining battery is easy to measure, the rate at which it will deplete is still uncertain. This uncertainty arises due to the fact that the energy spent by a forwarding node is proportional to the transmission power, i.e., the power at which a node transmits a packet so that the packet reaches the intended receiver. It is known that more power is required to communicate to a larger distance. Thus, transmit power depends on the relative distance between the transmitter and the receiver nodes. Note, that the maximum range $\left(R_{\max }\right)$ attainable by a node is limited by the maximum allowable transmit power, $P_{\max }$.

Let us now calculate the uncertainty in the relative distance between a transmitter and a receiver. Since, the nodes are randomly scattered, the receiver will lie anywhere in the circle with radius $R_{\max }$ with equal probability, with the transmitter node being at the center of the circle. If we use polar co-ordinates, the radial distance is assumed to be uniformly distributed between 0 and $R_{\max }$, and the angle uniformly distributed direction between 0 and $2 \pi$.

The position of the receiver is characterized by $f_{R}(r)$ and $f_{\Theta}(\theta)$, denoting respectively the distance probability density function ( $p d f)$ and the directional $p d f$. The two $p d f \mathrm{~s}$ are defined as follows:

$$
\begin{gathered}
f_{R}(r)= \begin{cases}\frac{2 r}{R_{\text {max }}^{2}}, & 0 \leq r \leq R_{\text {max }} \\
0, & \text { elsewhere. }\end{cases} \\
f_{\Theta}(\theta)= \begin{cases}\frac{1}{2 \pi}, & 0 \leq \theta \leq 2 \pi \\
0, & \text { elsewhere. }\end{cases}
\end{gathered}
$$

The joint $p d f$ is given by

$$
f_{R \Theta}(r, \theta)= \begin{cases}\frac{r}{\pi R_{\text {max }}^{2}}, & 0 \leq r \leq R_{\text {max }} \\ & 0 \leq \theta \leq 2 \pi \\ 0, & \text { elsewhere }\end{cases}
$$

Given this $p d f$ of the distance of the receiver from the transmitter, the transmission power distribution, and hence the energy dissipation can be obtained. For the joint $p d f$ of distance as $f_{R \Theta}(r, \theta)$, we calculate the $p d f$ for the transmission power. We assume that the attenuation in the signal strength is inversely proportional to the square of the distance i.e., if $P_{t}$ and $P_{r}$ are the transmit and receiver powers respectively,

$$
P_{r}=P_{t} \times d^{-\alpha}
$$

where $\alpha$ is the path loss exponent and usually lies between 2 and 6 . Therefore, the $p d f$ for the transmission power, $f_{P}\left(p_{t}\right)$, is given by

$$
f_{P}\left(P_{t}\right)= \begin{cases}f_{R \Theta}\left(P_{t}^{-\alpha}\right), & 0 \leq r \leq R_{\max } \\ & 0 \leq \theta \leq 2 \pi \\ 0, & \text { elsewhere }\end{cases}
$$

Since, according to our assumption transmission power is directly proportional to the energy consumed, we use the transmission power $p d f$ to calculate the energy entropy. We use Shannon's entropy for this purpose. Shannon's entropy for a random variable with $Y$ with $p d f f_{Y}(y)$ is

$$
H_{S}(Y)=\int_{-\infty}^{+\infty} f_{Y}(y) \log f_{Y}(y) d y
$$

Thus, the energy entropy is given by

$$
H\left(f_{P}\right)=\int_{0}^{R_{\max }}\left(f_{R \Theta}\left(P_{t}^{-\alpha}\right) \log f_{R \Theta}\left(P_{t}^{-\alpha}\right)\right) d P_{t}
$$

\section{Total Entropy}

At all times, every node computes its instantaneous mobility entropy $\left(H_{\text {mobility }}\right)$ and battery entropy $\left(H_{\text {energy }}\right)$ and announces these values to the current clusterhead (if it exists). The node with the lowest entropy wins the election; receives the node list for the virtual cluster and notifies each member of its new role. A node whose neighbor list never changes, would have a total combined entropy of 0 . A node with a significant amount of relative motion and a small residual battery would have significantly higher total entropy. We use a simple linear combination to find the total entropy, $H_{t o t a l}$. We define the total entropy as

$$
H_{\text {total }}=w_{1} H_{\text {mobility }}+w_{2} H_{\text {energy }}
$$

where $w_{1}$ and $w_{2}$ are the weighing factors and $w_{1}+w_{2}=1$. The weighting factors can be adjusted as per the desired priority for the network i.e., how important are mobility and energy with respect to each other.

\section{Simulation MODEl AND RESUltS}

To study the performance of our proposed clustering scheme, we conducted extensive simulation experiments where $N$ nodes were randomly distributed over an area of $100 \times 100$ units. The mobility of nodes followed the random waypoint model [5] with the displacement varying uniformly between 0 to a maximum value per unit time. Other parameters for simulation are shown in Table I.

To measure the performance of our proposed entropybased clustering algorithm, we identify three metrics: (i) the average number of clusterheads or clusters, (ii) the average number of clusterhead changes, and (iii) the average connectivity. It can be noted that the average size of a cluster, i.e., average number of nodes in a cluster is nothing but the total number of nodes in the system divided by the number of clusters. These three metrics are studied for the varying number of nodes, transmission range, and maximum displacement. 


\begin{tabular}{lll}
\hline Parameter & $:$ & Value \\
\hline$N$ & $:$ & $20,40,60,80,100$ \\
Max. displacemment & $:$ & $2-10$ \\
Transmission range & $:$ & $10-100$ \\
$w_{1}$ & $:$ & 0.5 \\
$w_{2}$ & $:$ & 0.5 \\
\hline
\end{tabular}

TABLE I

SIMULATION PARAMETERS

Figures 1 and 2 show the average number of clusters as a function of transmission range and maximum displacement respectively. For low transmission ranges, the number of clusters is much higher because the member nodes are likely to wander out of the range of the clusterhead. As the transmission range increases, nodes are more lileky to remain within the radio radius of the clusterhead regardless of how fast they move. As the average node velocity increases (increase in maximum displacement), there is a small increase in the number of clusters due to the nature of the motion to disperse the nodes.

Figures 3 and 4 show the average clusterhead connectivity, or nodal degree during the course of the simulation. As transmission ranges increase, there is almost a linear increase in the nodal degree, owing to the uniform random motion.

Figures 5 and 6 show the rate at which the clusterheads change. It is to be noted that a lower value of clusterhead changes is desirable since it reflects stability of the topology.

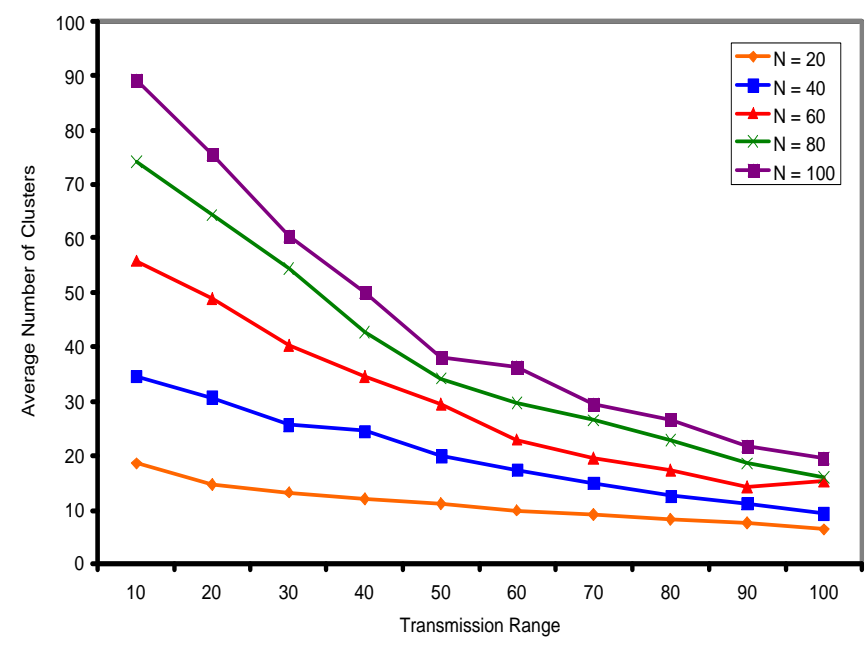

Fig. 1. Average number of clusters vs. transmission range

\section{CONCLUSIONS}

In this paper, we proposed a clustering scheme based on entropy measures. We considered two important aspects of ad hoc networks- mobility and energy consumption of nodes. Through the exchange of beacon messages, the nodes gather information about their mutual mobility and energy. We use a generic linear combination model to consider the both entropies. We conducted simulations that show the performance of the proposed clustering scheme in terms of

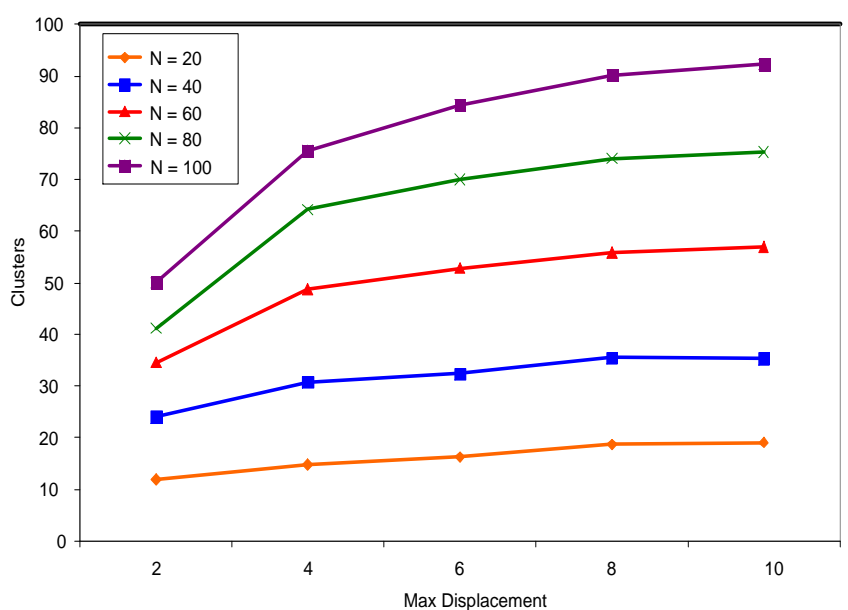

Fig. 2. Average number of clusters vs. maximum displacement

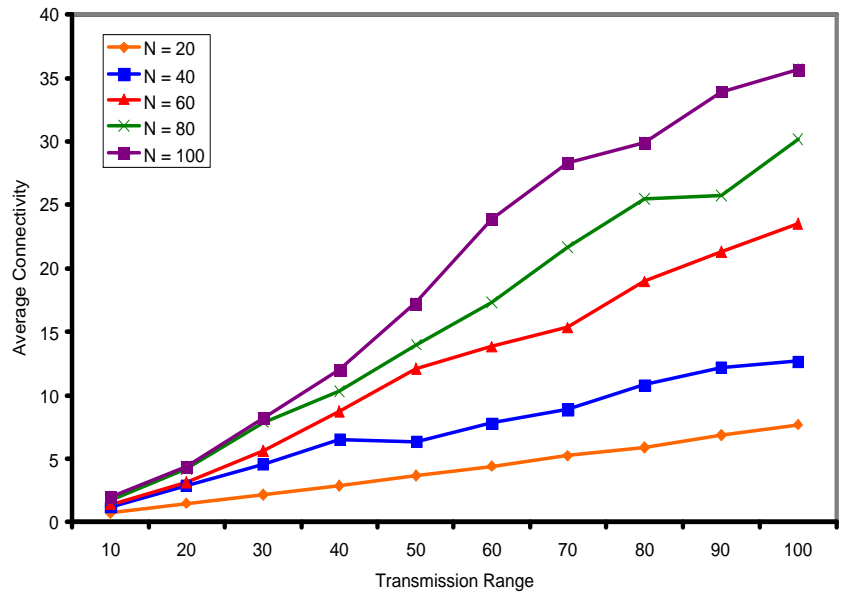

Fig. 3. Average connectivity vs. transmission range

the average number of clusters, the average number of cluster changes, and the average connectivity. We also compared our results to Lowest ID and Highest Degree clustering schemes. The results demonstrate that the mutual information captured through entropy is very effective in maintaining the stability of the network.

\section{REFERENCES}

[1] A. Amis and R. Prakash, "Load-balancing clusters in wireless ad hoc networks", Proc. of ASSET 2000, Richardson, TX, March 2000, pp. 25-32.

[2] D.J. Baker and A. Ephremides, "A distributed algorithm for organizing mobile radio telecommunication networks", Proc. of the 2nd Int. Conference on Distributed Computer Systems, April 1981, pp. 476483.

[3] D.J. Baker and A. Ephremides, "The architectural organization of a mobile radio network via a distributed algorithm", IEEE Transactions on Communications COM-29 11 (1981), pp. 1694-1701.

[4] S. Basagni, I. Chlamtac, and A. Farago, "A Generalized Clustering Algorithm for Peer-to-Peer Networks", Proc. of Workshop on Algo- 


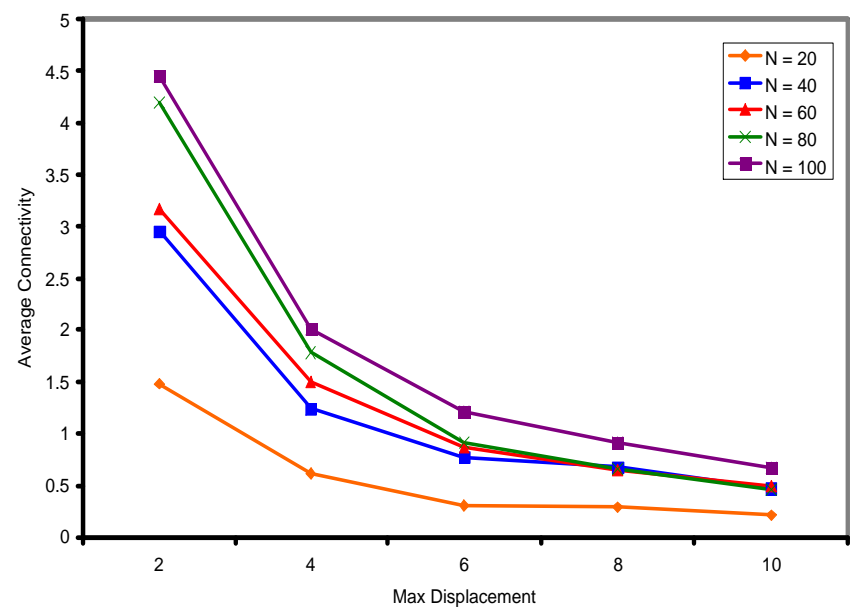

Fig. 4. Average connectivity vs. maximum displacement

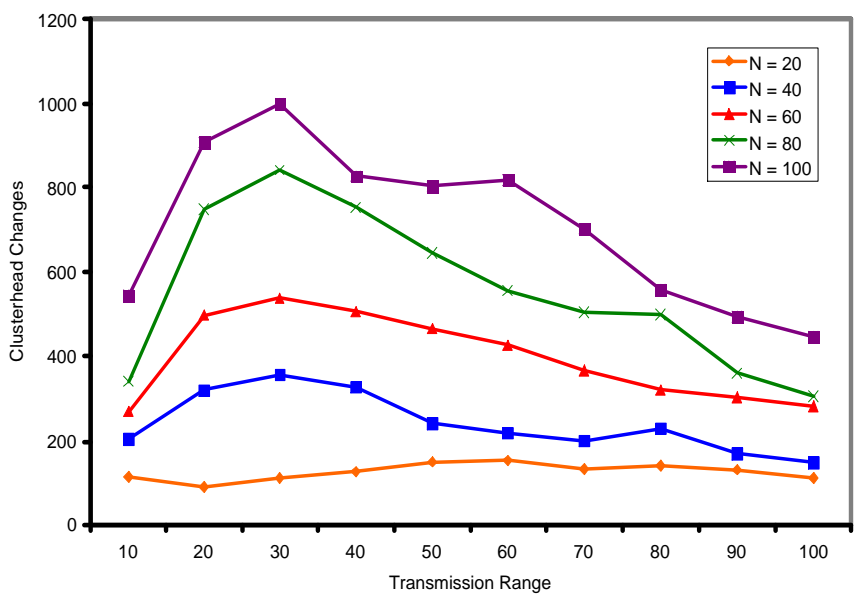

Fig. 5. Average clusterhead changes vs. transmission range

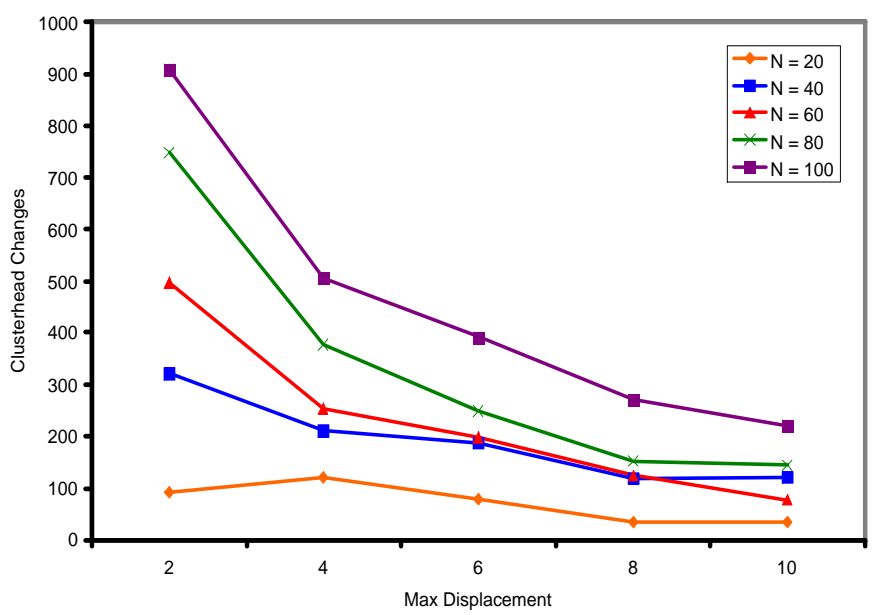

Fig. 6. Average clusterhead changes vs. maximum displacement rithmic Aspects of Communication (satellite workshop of ICALP), July 1997.

[5] C. Bettstetter, G. Resta, and P. Santi, "The node distribution of the random waypoint mobility model for wireless ad hoc networks", IEEE Transactions on Mobile Computing, Volume: 2, Issue: 3, September 2003, pp. 257-269.

[6] M. Chatterjee, S.K. Das and D. Turgut, "WCA: A Weighted Clustering Algorithm for Mobile Ad hoc Networks", Journal of Cluster Computing (Special Issue on Mobile Ad hoc Networks), Vol. 5, No. 2, April 2002, pp. 193-204.

[7] I. Chlamtac and A. Farago, "A New Approach to the Design and Analysis of Peer-to-Peer Mobile Networks", Wireless Networks, 5(3), August 1999, pp. 149-156.

[8] M. Gerla and J.T.C. Tsai, "Multicluster, mobile, multimedia radio network", Wireless Networks, Vol. 1, No. 3, 1995, pp. 255-265.

[9] A.B. McDonald and T.F. Znati, "A mobility-based framework for adaptive clustering in wireless ad hoc networks", IEEE Journal on Selected Areas in Communications, Vol. 17, No. 8, 1999, pp. 14661487.

[10] A.K. Parekh, "Selecting routers in ad-hoc wireless networks", Proceedings of the SBT/IEEE International Telecommunications Symposium, August 1994.

[11] D. Turgut, S.K. Das, R. Elmasri, B. Turgut, "Optimizing Clustering Algorithm in Mobile Ad hoc Networks Using Genetic Algorithmic Approach", Proc. of GLOBECOM 2002, Taipei, Taiwan, November $17-21,2002$

[12] D. Turgut, B. Turgut, R. Elmasri, Than V. Le, "Optimizing Clustering Algorithm in Mobile Ad hoc Networks Using Simulated Annealing", Proceedings of WCNC 2003, New Orleans, Louisiana, March 16-20, 2003 